\title{
Graves' ophthalmopathy: eye muscle involvement in patients with diplopia
}

\author{
Endre V Nagy, Judit Toth ${ }^{1}$, Ildiko Kaldi ${ }^{5}$, Judit Damjanovich ${ }^{2}$, Emese Mezosi, Agota Lenkey ${ }^{3}$ Laszlo Toth $^{4}$, \\ Jeno Szabo, Zsolt Karanyi and Andras Leovey \\ Departments of Medicine, ${ }^{1}$ Radiology, ${ }^{2}$ Ophthalmology, ${ }^{3}$ Clinical Chemistry and ${ }^{4}$ Otolaryngology, University of Debrecen Medical and \\ Health Science Center, and ${ }^{5}$ Ophthalmology Unit, Kenezy Hospital, Debrecen, Hungary \\ (Correspondence should be addressed to Endre V Nagy, Department I of Medicine, Medical and Health Science Center, University of Debrecen, \\ Debrecen Pf. 19, H-4012, Hungary; Email: nagy@ibel.dote.hu)
}

\begin{abstract}
Background: Diplopia identifies patients with eye muscle involvement in Graves' ophthalmopathy (GO). Objective: To identify clinical parameters that could eliminate the need for magnetic resonance imaging (MRI) to assess the activity of inflammation in the eye muscles of GO patients with diplopia. Methods: In 43 patients with GO with recently developed diplopia, orbital ultrasound and MRI were performed. Muscle diameters and MRI T2 relaxation times were measured, and the amount of orbital connective tissue was calculated from MRI scans and compared with ultrasound readings, diplopia grades, degree of protrusion, ocular pressure, tear production, antibody levels and hormonal parameters of thyroid function.

Results: No correlation was found between diameters of 233 extraocular muscles measured by MRI and by ultrasound. For each of the four muscles, there was a diameter above which ultrasound was always unreliable. MRI data were used in further analysis. Of the muscles examined, the inferior rectuses were the most frequently enlarged - at least one, in $93 \%$ of cases. Medial, lateral and superior rectuses were enlarged in $59 \%, 37 \%$ and $34 \%$ of the orbits respectively. The pattern of muscle involvement of the two orbits tended to be symmetric $(r=0.49, P=0.003)$, particularly for the medial rectuses $(r=0.90, P=0.000)$. Proptosis correlated with the sum of the muscle diameters for a given eye (right eye: $r=0.54, P=0.003$; left eye: $r=0.57, P=0.001$ ), but it failed to correlate with the amount of orbital connective tissue. In $53 \%$ of the patients, normal T2 relaxation times were found in all eight muscles. There was only a weak correlation between muscle thickness and $\mathrm{T} 2$ relaxation time $(r=0.49, P=0.003)$, indicating that muscle enlargement alone is not a sign of disease activity. The severity of diplopia was independent of $\mathrm{T} 2$ relaxation time. The amount of orbital connective tissue showed a negative correlation with the greatest T2 relaxation time for a given eye $(r=-0.52$, $P=0.004)$; this suggests that disease types exist that have predominant muscle involvement and predominant connective tissue expansion. No correlation between connective tissue expansion and proptosis, diplopia grade, muscle thickness or disease duration was found - that is, connective tissue expansion is not a major factor in diplopia. Both muscle and connective tissue findings were independent of thyroid function.

Conclusion: Ultrasound and MRI eye muscle diameter readings do not correlate, because of the inherent inaccuracy of orbital ultrasound. Muscle enlargement alone does not mean oedematous swelling and active disease. Neither ultrasound, nor any combination of 11 clinical and laboratory parameters provided the degree of information on muscles and connective tissue that was obtainable by MRI. In unclear cases of recently developed diplopia, before orbital decompression surgery, in the case of treatment failure or if, for any other reason, imaging is needed in GO, MRI is the method of choice.
\end{abstract}

European Journal of Endocrinology 142 591-597

\section{Introduction}

Eye protrusion in patients with Graves' ophthalmopathy (GO) is the result of extraocular muscle swelling, retroocular connective tissue expansion, or both (1-3). Diplopia is caused by external eye muscle dysfunction, either due to the presence of lymphocytic infiltration and edematous swelling (active disease) or fibrosis (inactive disease) in the muscles. It is essential to distinguish between active and inactive disease in the muscles for purposes of treatment decisions (4), as only patients with active disease will benefit from immunosuppressive treatment $(5,6)$. In contrast, patients with inactive disease are better candidates for 
muscle-corrective surgery because of the unpredictable results of corrective surgery in patients with signs of active inflammation in the muscles. Both active and inactive disease may present with enlarged muscles (7). Thus, in GO patients with diplopia, it is crucial to obtain reliable information to determine the type and degree of extraocular muscle involvement in order to institute appropriate treatment. In the present series of patients, using imaging techniques and clinical assessment of eye muscle and thyroid status, we failed to identify a combination of ultrasound and clinical data that could be substituted for MRI. In addition, we show that recentonset diplopia is not always accompanied by signs of active inflammation in the muscles.

\section{Patients and methods}

\section{Patients}

Forty-three patients with Graves' disease (age $45 \pm 11$ years, 37 women, six men) with GO were entered in the study. The selection criteria were the presence of diplopia that had developed within 90 days before presentation, and clinical and laboratory signs of existing Graves' disease, or a documented history of Graves' disease. In euthyroid antibody-negative patients without a known history of thyroid disease (one patient, see below), the minimum requirement was a less than normal response during thyrotrophinreleasing hormone (TRH) stimulation in the absence of any other eye or orbital disease that could explain the complaints. Patients with previously known Graves' disease had been treated earlier (drug, radioiodine or surgery) or were receiving continuous thyroid medication during the study. Nine of the 43 patients had already had ophthalmopathy for up to 5 years at the time of the first appearance of diplopia. All patients gave informed consent to participate in the study.

\section{Clinical assessment}

In patients complaining of diplopia, diplopia grade was assessed with the patient in a fixed-head position, asking the patient to follow (with the eyes only) a small tool (ballpen) that was moved from the centre of the binocular visual field towards its periphery in the four main directions. The severity of restriction of eye movement was graded as: $0=$ no diplopia (no patient had grade 0 in our series, see selection criteria above); $\mathrm{A}=$ diplopia only at extreme lateral and/or upward and/or downward gaze; $\mathrm{B}=$ diplopia in all but one single (usually forward) direction of gaze; $\mathrm{C}=$ diplopia in all directions of gaze. This severity score (ABC score) is slightly different from the diplopia score described in the 'no specs' classification of the Ad Hoc Committee of the American Thyroid Association (8).

Hess charts were constructed for each patient. Protrusion was measured with Hertel's exophthalmometer. Ocular pressure was measured using a tonometer. Tear production was estimated using Schirmer's test.

Thyroid hormone and thyroid-stimulating hormone (TSH) concentrations were measured using commercial methods (third generation TSH and free thyroxine $\left(\mathrm{FT}_{4}\right)$ : LIA-mat, Byk-Sangtec Diagnostica, Dietzenbach, Germany; free tri-iodothyronine $\left(\mathrm{FT}_{3}\right)$ : Abbott Laboratories, Abbott Park, IL, USA). TSH receptor binding antibodies (TRAb) were detected using the TRAK assay (Brahms, Berlin, Geremany).

\section{Imaging techniques}

Magnetic resonance images (MRI) of the orbits were performed using a Shimadzu SMT-100X (1.0 T) unit. Serial $3 \mathrm{~mm}$ coronal images, with $1 \mathrm{~mm}$ gaps, were taken of the medial, inferior and superior rectus muscles, and axial images of the lateral rectus muscles. After the horizontal diameters of the medial and lateral rectus muscles and the vertical diameters of the superior and inferior rectuses had been measured on the series of images, the largest diameter of the middle section of each muscle was chosen for further comparisons. T2 relaxation times were calculated and long T2 times were considered to indicate oedema $(7,9)$. The amount of orbital connective tissue was calculated from a series of axial images of the orbits taken parallel to the optic nerve with $3 \mathrm{~mm}$ slice thickness. From the images, the total volume of the bony orbit and the amount of orbital connective tissue were calculated, and the connective tissue volume $\left(\mathrm{mm}^{3}\right)$ :bony orbit volume $\left(\mathrm{mm}^{3}\right)$ ratio was regarded as the relative amount of connective tissue.

Orbital ultrasound (10) was performed by two independent ophthalmologists, using a NIDEK Echoscan 3300 with a $10 \mathrm{MHz}$ transducer. In addition to the routinely used B mode images, ophthalmologists were allowed to obtain simultaneous A mode scans if they felt that it helped to obtain more accurate measurement of muscle diameter.

\section{Statistical analysis}

Muscle diameters measured by MRI and ultrasonography were compared using Pearson's productmoment correlation test. Non-parametric comparisons were performed using the Kruskal-Wallis test or, where appropriate, the Mann-Whitney $U$ test. Twoby-two tables with Pearson's and Yates' $\chi^{2}$ calculations were analysed.

\section{Results}

\section{Patient characteristics and the functional status of the thyroid gland}

Seventy-seven percent of patients were between 30 and 60 years of age when diplopia appeared. At the 
Table 1 Correlation between extraocular muscle diameters obtained by ultrasound and by MRI.

\begin{tabular}{|c|c|c|c|c|c|c|}
\hline \multirow[b]{2}{*}{ Muscle } & \multicolumn{3}{|c|}{ Right eye } & \multicolumn{3}{|c|}{ Left eye } \\
\hline & Correlation & $P$ & $n$ & Correlation & $P$ & $n$ \\
\hline Inferior rectus & 0.0822 & 0.666 & 30 & 0.2794 & 0.142 & 29 \\
\hline Medial rectus & 0.0585 & 0.759 & 30 & 0.0722 & 0.699 & 31 \\
\hline Lateral rectus & -0.2049 & 0.296 & 28 & -0.1433 & 0.458 & 29 \\
\hline Superior rectus & 0.0408 & 0.837 & 28 & 0.3098 & 0.109 & 28 \\
\hline
\end{tabular}

time of the investigation, $29 \%$ of the patients were hyperthyroid, 56\% euthyroid and 15\% hypothyroid. The thyroid status represented the result of antithyroid treatment for Graves' disease in 33 patients. In ten of the 43 patients, diplopia was the presenting complaint that led to the diagnosis of Graves' disease. Before treatment, six were hyperthyroid, two had subclinical hyperthyroidism (defined as normal $\mathrm{FT}_{4}$, normal $\mathrm{FT}_{3}$ and subnormal TSH concentrations), one was euthyroid and one hypothyroid. The euthyroid patient, who presented with grade B diplopia, had no history of thyroid disease, and had no thyroid abnormalities except for a suppressed TSH response to TRH; on MRI, expansion of the retrobulbar connective tissue was observed. This patient's extraocular muscles were not enlarged, and $\mathrm{T} 2$ relaxation times were in the normal range.

\section{External eye muscle involvement}

The diplopia grades of the 43 patients examined were: grade A, 15 patients; grade B, 14 patients; grade C, 14 patients.

Readings of the diameters of 233 eye muscles obtained by MRI and ophthalmic ultrasonography were compared. Correlations for each muscle are shown in Table 1. Both sensitivity and specificity of ultrasound for the detection of enlarged muscles were low (sensitivities: inferior rectus 56.7\%, medial rectus $62.1 \%$, lateral rectus $52.8 \%$, superior rectus $65.6 \%$; specificities: inferior rectus $25.0 \%$, medial rectus $44.9 \%$, lateral rectus $43.6 \%$, superior rectus $24.0 \%$ ). In general, compared with results from MRI, those obtained by sonography underestimated muscle size,

Table 2 Normal extraocular muscle diameters as determined by MRI $(n=20)$.

\begin{tabular}{lc}
\hline Muscle & Diameter $(\mathrm{mm})$ \\
\hline Inferior rectus & $4.1 \pm 0.4$ \\
Medial rectus & $3.8 \pm 0.3$ \\
Lateral rectus & $3.1 \pm 0.2$ \\
Superior rectus & $3.5 \pm 0.2$ \\
\hline \multicolumn{2}{l}{ Values are means \pm SD. }
\end{tabular}

and many enlarged muscles were recorded as normal. For each muscle, there was a diameter (as determined by MRI) above which ultrasound was always unreliable (inferior rectus $12.5 \mathrm{~mm}$, medial rectus $9.0 \mathrm{~mm}$, lateral rectus $4.5 \mathrm{~mm}$, superior rectus $9.5 \mathrm{~mm}$ ). We used the MRI diameter readings in the investigations detailed below. The extraocular muscle diameters identified as normal by MRI, based on findings in 20 normal individuals, are shown in Table 2.

$\mathrm{T} 2$ relaxation times were considered indicative of increased water content (oedema) and active inflammation in the muscles if they were greater than $63 \mathrm{~ms}$ (mean \pm 2 s.D. of 20 healthy controls: $52.4 \pm 11.0 \mathrm{~ms}$ ). In 16 of 34 patients (47\%) at least one muscle had an increased $\mathrm{T} 2$ value. There was only a weak correlation between muscle thickness and $\mathrm{T} 2$ relaxation time ( $r=0.49, P=0.003)$, showing that muscle thickness alone is not a measure of the degree of inflammation. Diplopia grade did not predict inflammatory activity; Fig. 1 shows that there were equal chances of finding a normal or increased $\mathrm{T} 2$ relaxation time in all grades $(\mathrm{A}-\mathrm{C})$.

A tendency toward symmetry of the pattern of muscle involvement of the two eyes was observed in any individual patient $(r=0.52, P=0.001$ for all four muscles), particularly for the medial rectus muscles $(r=0.90, P=0.000)$. The number of patients with

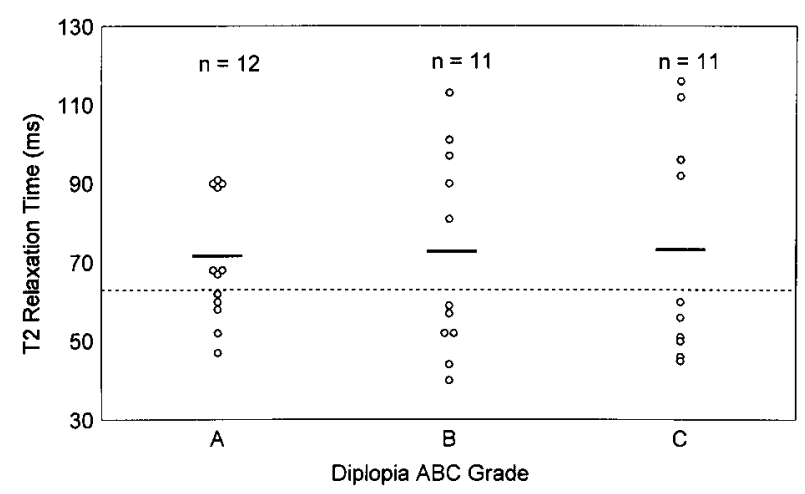

Figure 1 Activity of muscle inflammation in different diplopia grades. In all grades of diplopia there were nearly equal numbers of patients with normal and increased T2 relaxation times - that is, diplopia grade alone did not predict inflammatory activity. 


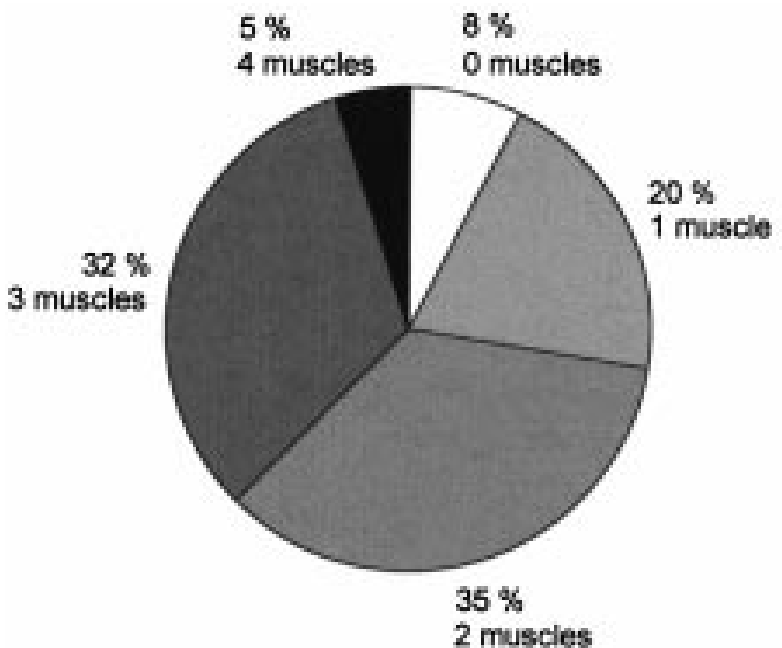

Right Eye

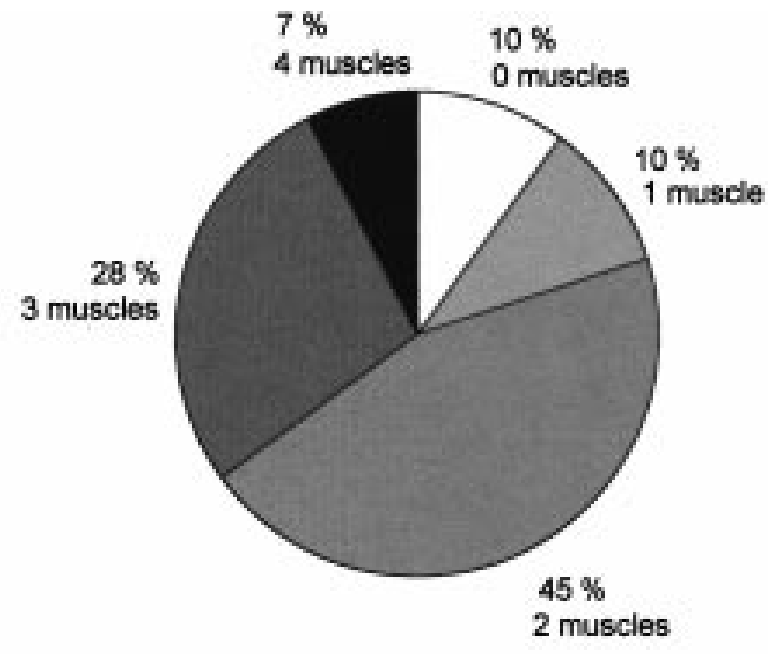

Left Eye

Figure 2 Numbers of enlarged muscles in patients with diplopia. Two or three muscles were involved in the majority of the patients.

one or more enlarged muscles is shown in Fig. 2. In two patients, no muscle enlargement and normal T2 relaxation times were identified on MRI. One of these two patients had no thyroid abnormality except for a suppressed TSH response to TRH, grade B diplopia and expanded orbital connective tissue. One year later, she had no diplopia and presented with subclinical hypothyroidism. The other patient had untreated Graves' disease, increased $\mathrm{FT}_{4}$ concentrations, normal amounts of orbital connective tissue and grade A diplopia. Both Hess charts were characteristic of mild bilateral medial rectus dysfunction, emphasizing the importance of clinical examination in addition to radiographic studies.

Among the extraocular muscles, the inferior rectus was the most frequently enlarged (Table 3 ). For the right and left eyes, this muscle was enlarged in 85 and $83 \%$ of the patients respectively; at least one of the two inferior rectus muscles was enlarged in all but three patients (93\%). Two of the three patients had no muscle involvement at all by MRI (see above), while one patient had a lone, mild medial rectus muscle

Table 3 Frequency of enlargement of extraocular muscles.

Frequency of enlargement (\%)

\begin{tabular}{lcc}
\cline { 2 - 3 } Muscle & Right eye & Left eye \\
\hline Inferior rectus & $85(n=40)$ & $83(n=42)$ \\
Medial rectus & $65(n=40)$ & $83(n=41)$ \\
Superior rectus & $43(n=35)$ & $30(n=37)$ \\
Lateral rectus & $32(n=38)$ & $36(n=39)$ \\
\hline
\end{tabular}

enlargement. If two or more muscles were affected in a patient, the inferior rectus muscle was always one of them.

\section{Orbital connective tissue}

In 20 normal orbits, the connective tissue:bony orbit ratio was $0.47 \pm 0.02$; that for the patients' eyes was $0.59 \pm 0.10$. There was a negative correlation between the amount of connective tissue and the $\mathrm{T} 2$ relaxation time of the most severely involved muscle in the orbit $(r=-0.52, P=0.004)$. This means that patients with more connective tissue were less likely to have inflammatory signs in the muscles. No correlation between connective tissue status and individual or cumulative (sum of muscle diameters for a given eye) muscle diameters, diplopia $\mathrm{ABC}$ grade, proptosis, ocular pressure, patient age, thyroid function or TRAb levels was found.

\section{Hess charts}

Hess charts showed axis deviation in all cases. However, neither the number of involved muscles, nor the muscles themselves could be correctly identified on the basis of these charts, by an investigator blinded to the results of MRI and ultrasound.

\section{Proptosis, intraocular pressure, tear production}

For the entire group of patients with diplopia, the mean Hertel readings $(20.5 \pm 3.7 \mathrm{~mm}$ right eye, $20.0 \pm$ $4.0 \mathrm{~mm}$ left eye; upper limit of normal based on the 
mean +2 s.D. of 50 Hungarian individuals, $17.0 \mathrm{~mm}$ ) showed exophthalmos. Correlation was found between the degree of exophthalmos and the sum of muscle diameters on MRI for a given eye (right eye: $r=0.54$, $P=0.003$; left eye: $r=0.57, P=0.001)$. Of the diameters, those of the right and left medial rectuses and left superior rectus correlated with Hertel readings ( $r$ values $0.40,0.50$ and 0.46 respectively; $P<0.05$ for each muscle). However, a normal Hertel reading did not guarantee normal sized muscles on MRI. No correlation between the amount of orbital connective tissue and the degree of proptosis was found. Interestingly, a negative correlation was noted between the degree of exophthalmos and the diplopia $A B C$ grade: Hertel values were consistent with more severe exophthalmos in the presence of grade A diplopia than in B or C diplopia (right eye: grade A $22.3 \pm 3.7 \mathrm{~mm}$, grade B $19.9 \pm 3.2 \mathrm{~mm}$, grade $\mathrm{C}, 18.0 \pm 2.7 \mathrm{~mm}$; left eye: grade A, $21.7 \pm 4.1 \mathrm{~mm}$, grade $\mathrm{B}, 19.8 \pm 3.1 \mathrm{~mm}$, grade $\mathrm{C}, 17.4 \pm 3.0 \mathrm{~mm}$; correlations between Hertel readings and diplopia $\mathrm{ABC}$ grades: $r=-0.49$, $P=0.004$ and $r=-0.47, P=0.007$ for the right and left eyes respectively).

Mean ocular pressure $(19.2 \pm 3.78 \mathrm{mmHg})$ was near the upper limit of normal and mean tear production $(13.23 \pm 8.0 \mathrm{~mm}$ in $5 \mathrm{~min})$ was near the lower limit of normal. Individual values were independent of muscle and connective tissue status. Neither the functional status of the thyroid nor the presence or absence of TRAb influenced tear production.

\section{Thyroid function tests and TSH receptor antibodies}

No correlation was found between thyroid function (TSH, $\mathrm{FT}_{4}, \mathrm{FT}_{3}$ ) and any of the other parameters studied.

The TRAb level for the whole group was $22.2 \pm$ $24.4 \mathrm{U} / \mathrm{l}$ (normal less than 15 U/l). TRAb levels correlated with the diameters of medial rectus muscles (right: $r=0.58, P=0.009$, left $r=0.48, P=0.043$ ); no correlation with any of the other muscles was found. TRAb did not correlate with diplopia $A B C$ grade, proptosis, ocular pressure, tear production, T2 relaxation time, or the amount of orbital connective tissue.

\section{Discussion}

External eye muscles are important contributors to the orbital process in Graves' ophthalmopathy. Our cohort of patients was composed of individuals who developed diplopia within 90 days of enrolment in the study. The rather unusual selection criterion, diplopia, guaranteed that muscle dysfunction was present and allowed us to describe characteristics of the affected extraocular muscles. The age distribution of our patients with diplopia was somewhat different from that previously described for ophthalmopathy (11); in our group, few patients were younger than 30 years and the ages of the majority of our patients were clustered around the fifth decade.

For clinical assessment of the diplopia at presentation, a three-grade system was used. The system, termed $\mathrm{ABC}$ diplopia score, is simple and interobserver variation is minimal. In common with the 'no specs' (8) and other scoring systems, the $\mathrm{ABC}$ score also requires more accurate supplementary techniques to detect and follow fine changes in restricted eye movements. Approximately equal numbers of patients were found in the three severity groups (A, B and C).

Nearly $50 \%$ of the patients had normal $\mathrm{FT}_{4}$ and $\mathrm{FT}_{3}$ concentrations when diplopia developed. This high number is explained by the fact that many patients (33 of 43) had been treated earlier or had been taking drugs for hyperthyroid Graves' disease at the time of investigation. In contrast, the majority of the ten patients in whom diplopia was the presenting complaint were hyperthyroid.

Both MRI (9) and ultrasound (12-14) are widely used for the detection and follow-up of muscle involvement in GO. For reasons of cost, it would be helpful to identify those patients who may be evaluated by ultrasound and those who need MRI. We were unable to identify clinical parameters that could predict the unsuitability of a given patient for sonographic muscle diameter measurement on admission. The apparent inaccuracy of ophthalmic ultrasound in patients with diplopia obliged us to use MRI diameter readings for comparisons and statistics. On the basis of the present results, which are consonant with others' (15), we do not recommend ultrasound as the first approach, because of variability in orbital tissue measurements. With the combined use of MRI and ultrasound, one can decide if ultrasound is sufficient for follow-up or if repeated MRI is required. MRI is also crucial for the measurement of $\mathrm{T} 2$ relaxation time, which helps to estimate the activity of inflammation in the muscles $(4,16)$. Extremely enlarged muscles were especially liable to be underscored by ultrasound: for each muscle, there was a diameter (as determined by MRI) above which ultrasound was always unreliable. One reason for this, among others (17), is that MRI affords the possibility of finding the thickest section of the muscle, whereas ultrasound is limited by examination angles. The lack of muscle enlargement or extended T2 on MRI in two of the patients with diplopia may mean that MRI is not sensitive enough to detect early muscle changes that cause muscle dysfunction. Alternatively, there may be mechanisms of muscle dysfunction other than those that lead to muscle thickening or oedematous swelling.

Of the muscles examined, at least one of the inferior rectuses was involved in $93 \%$ of the patients. The frequency of the involvement of each muscle was comparable to the findings of three other groups 
$(18-20)$, in spite of the exclusion of GO patients with no diplopia from our series. Muscle thickness correlated only weakly with the activity of disease estimated from the $\mathrm{T} 2$ relaxation time. Although enlarged muscles were more likely to occur with longer T2 relaxation times, muscle enlargement also occurred independently of T2 relaxation time. Similarly, severe (grade B or C) diplopia was found in equal number with normal and abnormal T2 relaxation times. Neither thick muscle(s) nor severe diplopia is equivalent to active inflammation in the muscles; T2 relaxation time measurements or other supplementary techniques, such as glycosaminoglycan determination in the urine (21), are needed to assess muscle status.

The symmetric MRI muscle enlargement patterns of the eyes suggest that the autoimmune process is symmetric, and other local factors may account for any asymmetric appearance and clinical parameters. Local treatments, including radiation therapy, should target both eyes, regardless of the often non-symmetric clinical appearance.

The negative correlation between $\mathrm{T} 2$ relaxation time in the muscles and the amount of connective tissue suggests that disease types exist that have predominant muscle involvement and predominant connective tissue expansion. We speculate that two components of the immune process act on different tissues - muscles and connective tissue. Expanded connective tissue is not a major factor in diplopia, because diplopia $\mathrm{ABC}$ grade did not correlate with connective tissue status, and because proptosis was not dependent on the amount of connective tissue.

We found a correlation between the degree of exophthalmos and the sum of muscle diameters for a given eye. The thickness of the medial rectuses also correlated with Hertel readings. A similar connection between exophthalmos and computer tomographic volumetric readings has been described by others (22). However, in our series a normal Hertel did not guarantee normal sized muscles. The radiographic diameter of the medial rectus muscle on MRI correlated with TRAb. Although others have described an even stronger connection between TRAb levels and GO (23; for review, see 24), the correlation in the present study was weak and may not be clinically relevant.

These results show that when diplopia manifests during the course of GO, imaging by MRI reveals at least one enlarged muscle in the majority of patients (in $95 \%$ in this series). Muscle water content, as judged by $\mathrm{T} 2$ relaxation time, varied from normal to severe inflammation. Ultrasound alone or in combination with any clinical or laboratory parameters was not sufficient, and often misleading, in the assessment of muscle status. In unclear cases of recently developed diplopia, before orbital decompression surgery, in the case of treatment failure or if, for any other reason, imaging is needed in GO, MRI is the method of choice.

\section{Acknowledgements}

We thank Dr Matthew S Ringel for critical review of this manuscript.

\section{References}

1 Riley FC. Orbital pathology in Graves' disease. Mayo Clinic Proceedings 197247 975-979.

2 Trokel L \& Jakobiec FA. Correlation of CT scanning and pathologic features of ophthalmic Graves' disease. Ophthalmology 198188 553-564.

3 Hosten N, Lietz A, Noske W \& Bechrakis NE. Endokrine orbitopathie. Fortschritte Röntgenstrahlen 1993159 304-306.

4 Utech CI, Khatibnia U, Winter PF \& Wulle KG. MR T2 relaxation time for the assessment of retrobulbar inflammation in Graves' ophthalmopathy. Thyroid 19955 185-193.

5 Prummel MF, Mourits MP, Berghout A, Krenning EP, Gaag R, Koorneef L \& Wiersinga WM. Prednisone and cyclosporine in the treatment of severe Graves' ophthalmopathy. New England Journal of Medicine 1989321 1353-1359.

6 Just M, Kahaly G, Higer HP, Rosler HP, Kutzner J, Beyer J et al. Graves' ophthalmopathy: role of MR imaging in radiation therapy. Radiology 1991179 187-190.

7 Pedrosa P, Pfannenstiel P, Just M, Higer HP, Utech C, Brederfoff J et al. Results of nuclear magnetic resonance tomography studies in endocrine orbitopathy. Klinische Monatsblatter für Augenheilkunde 1988193 169-173.

8 Werner SC. Modification of the classification of the eye changes of Graves' disease: recommendations of the Ad Hoc Committee of the American Thyroid Association. Journal of Clinical Endocrinology and Metabolism 197744 203-204.

9 Hosten N, Sander B, Cordes M, Schubert C, Schörner W \& Felix R. Graves' ophthalmopathy; MR imaging of the orbits. Radiology $1989172759-762$.

10 Werner SC, Coleman DJ \& Franzen LA. Ultrasonographic evidence of a consistent orbital involvement in Graves' disease. New England Journal of Medicine 1974290 1447-1450.

11 Bartley GB, Fatourechi V, Kadrmas EF, Jacobsen SJ, Ilstrup DM, Garrity JA et al. The chronology of Graves' ophthalmopathy in an incidence cohort. American Journal of Ophthalmology 1996121 426-434.

12 Given-Wilson R, Pope RM, Michell MJ, Cannon R \& McGregor AM. The use of real/time orbital ultrasound in Graves' ophthalmopathy: a comparison with computer tomography. British Journal of Radiology 198962 705-709.

13 Prummel MF, Suttorp-Schulten MSA, Wiersinga WM, Verbeek AM, Mourits MP \& Koornneef L. A new ultrasonographic method to detect disease activity and predict response to immunosuppressive treatment in Graves' ophthalmopathy. Ophthalmology 1993100 556-561.

14 Erickson BA, Harris GJ, Lewandowski MF, Murray KJ \& Massaro BM. Echographic monitoring of response of extraocular muscles to irradiation in Graves' ophthalmopathy. International Journal of Radiation Oncology Biology Physics 199531 651-660.

15 Demer JL \& Kerman BM. Comparison of standardized echography with magnetic resonance imaging to measure extraocular muscle size. American Journal of Ophthalmology 1994118 351-361.

16 Pauleit D, Schuller H, Textor J, Leutner C, Keller E, Sommer T et al. MR relaxation time measurements with and without selective fat suppression (SPIR) in endocrine orbitopathy. Rofo, Fortschritte auf dem Gebiete Rontgenstrahlen und der Neuen Bildgebenden Verfahren $1997176557-564$.

17 Gorman CA. The measurement of change in Graves' ophthalmopathy. Thyroid 19988 539-543.

18 Markl AF, Hilbertz T \& Mann K. Graves' ophthalmopathy: standardized evaluation of computed tomography evaluations; magnetic resonance imaging. Developments in Ophthalmology $19892038-50$ 
19 Delint PJ, Mourits MP, Kerlen CH, Scheenloop JJ \& Wittebol-Post D. B-scan ultrasonography in Graves' orbitopathy. Documenta Ophthalmologica 199385 1-4.

20 Wiersinga WM, Smit T, van der Gaag Mourits M \& Koornneef L. Clinical presentation of Graves' ophthalmopathy. Ophthalmic Research 198921 73-82.

21 Kahaly G, Forster G \& Hansen C. Glycosaminoglycans in thyroid eye disease. Thyroid 19988 429-432.

22 Hallin ES \& Feldon SE. Graves' ophthalmopathy: II. Correlation of clinical signs with measures derived from computed tomography. British Journal of Ophthalmology 198872 678682.
23 Nishikawa M, Yoshimura M, Toyoda N, Masaki H, Yonemoto T, Gondou A et al. Correlation of orbital muscle changes evaluated by magnetic resonance imaging and thyroid stimulating antibody in patients with Graves' ophthalmopathy. Endocrinology 1993 129 213-219.

24 Burch HB \& Wartofsky L. Graves' ophthalmopathy: current concepts regarding pathogenesis and management. Endocrine Reviews 199314 474-493.

Received 21 July 1999

Accepted 9 February 2000 